\title{
Downsizing and Silicon Integration of Photoacoustic Gas Cells
}

\author{
A. Glière ${ }^{1}$ (D) $\cdot$ P. Barritault ${ }^{1} \cdot$ A. Berthelot ${ }^{1} \cdot$ C. Constancias ${ }^{1} \cdot$ J.-G. Coutard ${ }^{1}$. \\ B. Desloges ${ }^{1} \cdot$ L. Duraffourg ${ }^{1} \cdot$ J.-M. Fedeli ${ }^{1} \cdot$ M. Garcia ${ }^{1} \cdot$ O. Lartigue ${ }^{1}$. \\ H. Lhermet ${ }^{1} \cdot$ A. Marchant ${ }^{1} \cdot$ J. Rouxel ${ }^{1,2} \cdot$ J. Skubich $^{1,3} \cdot$ A. Teulle ${ }^{1} \cdot$ T. Verdot $^{1}$. \\ S. Nicoletti ${ }^{1}$
}

Received: 6 September 2019 / Accepted: 29 November 2019 / Published online: 8 January 2020 (c) The Author(s) 2020

\begin{abstract}
Downsizing and compatibility with MEMS silicon foundries is an attractive path towards a large diffusion of photoacoustic trace gas sensors. As the photoacoustic signal scales inversely with the chamber volume, a trend to miniaturization has been followed by several teams. We review in this article the approach initiated several years ago in our laboratory. Three generations of components, namely a $40 \mathrm{~mm}^{3}$ 3D-printed cell, a $3.7 \mathrm{~mm}^{3}$ silicon cell, and a $2.3 \mathrm{~mm}^{3}$ silicon cell with a builtin piezoresistive pressure sensor, have been designed. The models used take into account the viscous and thermal losses, which cannot be neglected for such smallsized resonators. The components have been fabricated either by additive manufacturing or microfabrication and characterized. Based on a compilation of experimental data, a similar sub-ppm limit of detection is demonstrated. All three versions of photoacoustic cells have their own domain of operation as each one has benefits and drawbacks, regarding fabrication, implementation, and ease of use.
\end{abstract}

Keywords MEMS · Photoacoustic spectroscopy · Silicon integration · Trace gas measurements

This article is part of the selected papers presented at the 20th International Conference on Photoacoustic and Photothermal Phenomena.

A. Glière

alain.gliere@cea.fr

1 CEA, LETI, Univ. Grenoble Alpes, 38000 Grenoble, France

2 Present Address: IFREMER, LDCM, Centre de Bretagne, 29280 Plouzané, France

3 Present Address: GO Concept Alpes, 38000 Grenoble, France 


\section{Introduction}

Integration on silicon platform led in the recent years to major successes in the domain of micro-electromechanical systems (MEMS). The cost reduction reached through mass production in MEMS silicon foundries has for instance enabled large-scale deployment of accelerometers in the automotive industry or capacitive microphones in cellular phones [1]. The transposition of this strategy to photoacoustic (PA)-based trace gas sensors has been the object of a noteworthy effort in our laboratory, aiming at a widespread usage in indoor and outdoor air quality measurement. The ultimate goal is to develop a highly sensitive gas sensor combining, on the same chip, a mid-infrared laser, a light injection circuit, and a PA cell including an integrated pressure sensor. The scope of this paper is limited to the latter building block, namely the PA cell.

As the photoacoustic signal is inversely proportional to the chamber volume [2], a straightforward way to improve the sensor resolution consists in reducing its size. This trend towards miniaturization which, when pushed to limits, faces some limitations [3], has been followed by several teams during the last decades [4]. We review hereunder the existing literature on miniaturized sensors from two different perspectives towards cost reduction: (i) additive manufacturing, nowadays limited to small-scale production, and (ii) silicon microfabrication in MEMS foundries, well adapted to high-volume production.

On the one hand, the 3D-printing approach has first been adopted a few years ago by Bauer et al. [5] and more recently by Rück et al. [6]. In both cases, the PA cell was built with a 3D-printed polymer chamber and equipped with a MEMS microphone. The light was produced by an external laser diode, respectively, in the near-infrared or in the visible range. At the University of Freiburg, Palzer et al. intend to build an entire sensor, including a mid-infrared LED source, compatible with integration in a smartphone, at the expense of reduced performances [7]. Their measurement principle relies on a combination of absorption spectroscopy and photoacoustics. The absorption chamber is hot-embossed in polymethylmethacrylate (PMMA). The PA chamber is filled with the gas of interest (carbon dioxide or methane, used as a perfectly matched filter) and equipped with a MEMS microphone.

On the other hand, relying on silicon microfabrication techniques has first been considered, more than 20 years ago, by Weber et al. [8]. They imagined, but apparently never realized, a complete sensor composed of a filtered blackbody infrared source, a PA chamber, and a suspended membrane, detecting the pressure fluctuations by capacitive or piezoelectric means. A few years later, the same principle was successfully implemented by Ledermann et al. [9]. They combined a commercial micromachined infrared light source, filtered to match the $4.2 \mu \mathrm{m}$ absorption band of $\mathrm{CO}_{2}$, and a piezoelectric thin film deposited on a silicon suspended cantilever. During the same period, Firebaugh et al. [10] developed a PA cell built with two bonded silicon wafers. The first wafer was dedicated to the PA chamber, the fluidic supply, and the light port. The second one, a SOI wafer, featured a silicon membrane, used as a microphone by the means of a fiber optic 
displacement sensor. In a subsequent work [11], a third wafer was added and the optical displacement sensor was replaced by a piezoelectric one. The same team later abandoned the challenging multi-wafer stack option in favor of traditional machining techniques to fabricate a MEMS scale sensor [12].

In this paper, we review the PA cells downsizing approach that has been initiated several years ago at CEA-LETI. The timeline has been marked by the design, fabrication, and characterization of three generations of miniature photoacoustic components, namely a $40 \mathrm{~mm}^{3}$ 3D-printed metal cell [13], a $3.7 \mathrm{~mm}^{3}$ silicon cell [14], and a $2.3 \mathrm{~mm}^{3}$ silicon cell embedding its own piezoresistive pressure sensor [15]. The whole research and development process is presented and new experimental results are introduced. A concise presentation of the different PA set-ups is presented in Table 1 , in the concluding section.

\section{Miniaturized Differential Helmholtz Resonator}

The two first generations of PA cells rely on the differential Helmholtz resonator (DHR) architecture [16], composed of two identical chambers linked by two capillaries (Fig. 1). The gas inlet and outlet are connected to the middle of the capillaries. Photoacoustic excitation is ensured by lighting one of the chambers with a laser source. Although only one chamber is illuminated, the acoustic waves, opposite in phase at the Helmholtz resonance frequency, are generated in both chambers and the output signal consists in the difference of the measurements provided by two microphones, each one sensing the pressure in one chamber. This differential pressure measurement strategy provides a partial immunity to external acoustic noise.

\subsection{D-printed Cell}

The acoustic model used for the design of the miniaturized cells has already been presented elsewhere [17] and only the main features are reminded in this paper. The

Table 1 Summary of the performances reached by the three generations of miniaturized PA cells

\begin{tabular}{llllcl}
\hline Experiment & Source & Cell & Int. time (s) & LOD $_{1 \sigma}(\mathrm{ppb})$ & $\begin{array}{l}\mathrm{NNEA}_{1 \sigma}\left(\mathrm{cm}^{-1} \cdot \mathrm{W} /\right. \\
\left.\mathrm{Hz}^{1 / 2}\right)\end{array}$ \\
\hline $\mathrm{CH}_{4}, 2979 \mathrm{~cm}^{-1}$ & ICL, 2.2 mW & 3D steel & 2.7 & 126 & $9.110^{-9}$ \\
$\mathrm{H}-\mathrm{CHO}, 1765 \mathrm{~cm}^{-1}$ & QCL, 80 mW & 3D steel & 0.9 & 22 & $2.010^{-8}$ \\
$\mathrm{NO}, 1906.1 \mathrm{~cm}^{-1}$ & QCL, $120 \mathrm{~mW}$ & 3D steel & 7.0 & 7 & $2.310^{-8}$ \\
$\mathrm{NO}, 1906.1 \mathrm{~cm}^{-1}$ & QCL, $100 \mathrm{~mW}$ & 3D PMMA & 0.2 & 650 & $6.310^{-8}$ \\
$\mathrm{CF}_{4}, 1283 \mathrm{~cm}^{-1}$ & QCL, $67 \mathrm{~mW}$ & microfab. Si & 1.0 & 18 & $2.210^{-6}$ \\
$\mathrm{CO}, 2127.7 \mathrm{~cm}^{-1}$ & QCL, 75 mW & microfab. Si & 2.1 & 420 & $2.010^{-6}$ \\
$\mathrm{CH}_{4}, 3058 \mathrm{~cm}^{-1}$ & ICL, $2.5 \mathrm{~mW}$ & microfab. Si & 1.0 & 170 & $1.610^{-8}[14]$ \\
$\mathrm{NO}_{1} 1903.1 \mathrm{~cm}^{-1}$ & QCL, $123 \mathrm{~mW}$ & microfab. Si & 0.2 & 89 & $1.510^{-8}$ \\
$\mathrm{CO}_{2}, 2302.7 \mathrm{~cm}^{-1}$ & QCL, $4.9 \mathrm{~mW}$ & integrated Si & 7.0 & 900 & $3.010^{-7}$ \\
$\mathrm{CH}_{4}, 2979 \mathrm{~cm}^{-1}$ & ICL, $1.8 \mathrm{~mW}$ & integrated Si & 7.0 & 2500 & $2.410^{-7}$ \\
\hline
\end{tabular}




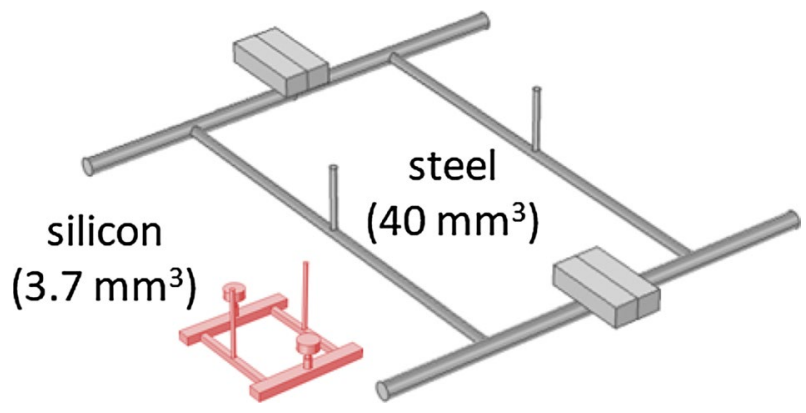

Fig. 1 Sketch of the two generations of DHR cells (gray: 3D-printed cell, red: microfabricated cell)

calculations are based on the resolution of the harmonic version of the Full Linearized Navier-Stokes (FLNS) coupled equations system, composed of the linearized versions of the momentum, mass, and energy conservation equations. Unlike the simplified acoustic wave equation, this framework enables handling losses of viscous or thermal origin. Indeed, in the useful 1 to $20 \mathrm{kHz}$ acoustic frequency range of interest to us, dissipative phenomena cannot be neglected because the viscous and thermal boundary layers, spanning on several tens of micrometers in air, occupy a significant proportion of the volume of the narrowest components of the acoustic network composing the photoacoustic cell.

The resolution is performed, by the finite element method (FEM), with the commercial software COMSOL Multiphysics (Comsol AB, Stockholm, Sweden). The finite element mesh of the study domain is refined in the vicinity of the walls so that several elements, usually three, are placed across the thickness of the viscous boundary layer. The heat source is obtained by the Beer-Lambert exponential attenuation law, assuming a perfectly collimated illumination of the entrance optical window. The cell is filled with air at standard temperature and pressure, whose physical properties are extracted from the material database of the software. Regarding boundary conditions, all walls are considered rigid and isothermal. The gas inlet and outlet are subject to atmospheric pressure and are not the site of any heat exchange. The pressure signal is defined as the average, on the microphone membrane, of the amplitude of the pressure field.

In this design, all the cavities are cylindrical. The diameter and length of the chamber are $750 \mu \mathrm{m}$ and $20 \mathrm{~mm}$, and those of the capillaries are $500 \mu \mathrm{m}$ and $20 \mathrm{~mm}$. The expected resonance frequency is $2.3 \mathrm{kHz}$, which is close to the $2.2 \mathrm{kHz}$ measured one.

The cell, made of stainless steel, is 3D-printed by direct metal laser sintering. The cell hosts a pair of commercial top port capacitive MEMS microphones (Fig. 2), directly glued on the metal block. The output analogical signal is processed by an interface discrete electronic circuit placed on a PCB.

Various measurements, based on set-ups involving slightly different versions of the 3D-printed cell, capacitive microphones and readout configurations, and addressing several trace gases, consistently lead to Normalized Noise Equivalent Absorption (NNEA) values in the order of $10^{-8} \cdot \mathrm{cm}^{-1} \cdot \mathrm{W} / \mathrm{Hz}^{1 / 2}$ (Table 1). 

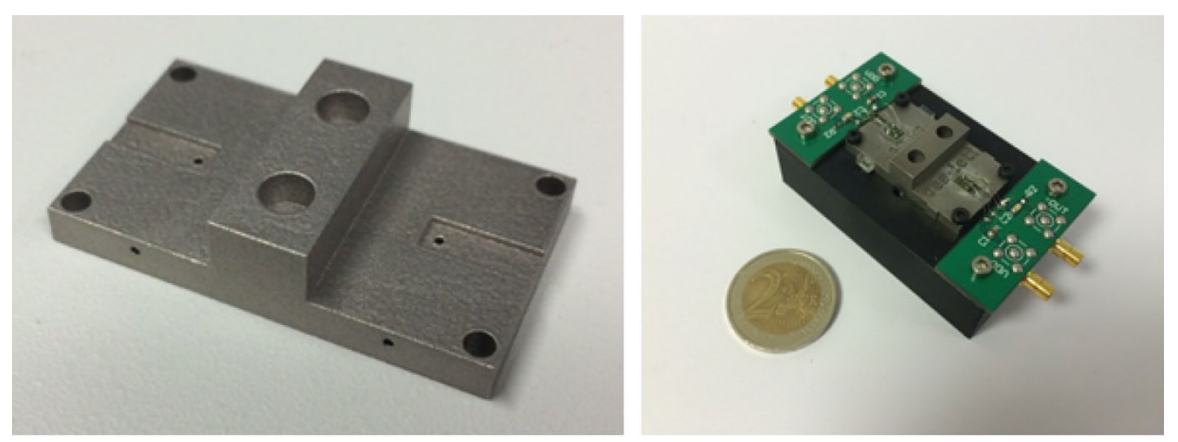

Fig. 2 Picture of the 3D-printed photoacoustic cell (left) and of the sensor mounted on its base and equipped with microphones and PCB (right). The metal block outer dimensions are $2 \mathrm{~cm} \times 3.5 \mathrm{~cm}$

Several examples of trace gas measurements are presented. In the first set-up, an interband cascade laser (Nanoplus, Gerbrunn, Germany) was adjusted on the $2979 \mathrm{~cm}^{-1}$ absorption line of methane [13]. The methane concentration in nitrogen was varied between $1 \mathrm{ppm}$ and $2000 \mathrm{ppm}$. The $2.2 \mathrm{~mW}$ laser power was modulated in amplitude and the integration time was fixed to $2.7 \mathrm{~s}$. The metal cell was equipped with transparent barium fluoride windows. A linear regression was then performed on the measurements, leading to an estimated limit of detection $\mathrm{LOD}_{1 \sigma} \approx 126 \mathrm{ppb}$. An additional measurement, made with a $0.5 \mathrm{ppm}$ dilution, confirmed the ability of the component to detect sub-ppm concentrations (Fig. 3).

A second set of experiments was performed with a mixture of formaldehyde in nitrogen. The set-up involved a $80 \mathrm{~mW}$ quantum cascade laser (QCL) (mirSense, Palaiseau, France) tuned on the $1765 \mathrm{~cm}^{-1}$ peak of formaldehyde. The cell was equipped with sapphire windows. The gas mixture was injected, from a gas diluter developed in our laboratory, in a range from $0 \mathrm{ppm}$ to $10 \mathrm{ppm}$, by steps of $1 \mathrm{ppm}$ (Fig. 4). The QCL current, and thus the optical wavelength, was modulated to carry

Fig. 3 Measurement of the PA signal for $0.5 \mathrm{ppm}$ of methane in nitrogen or $\approx 1.8 \mathrm{ppm}$ of methane in ambient air

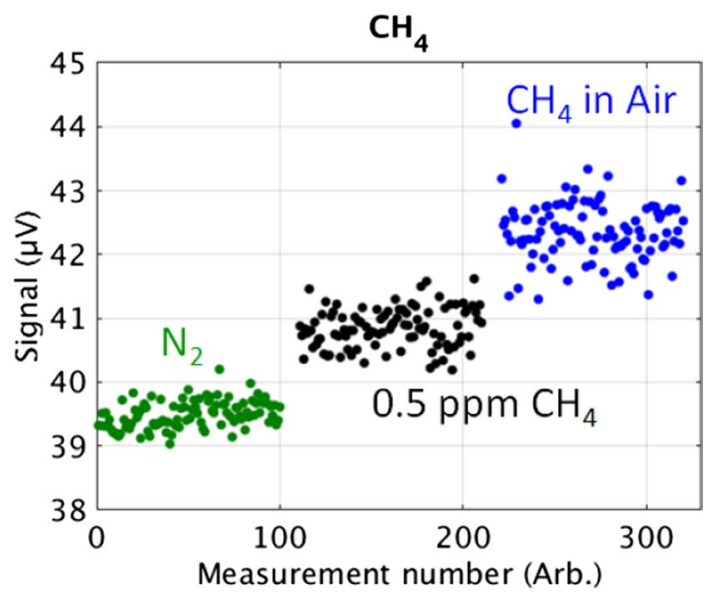



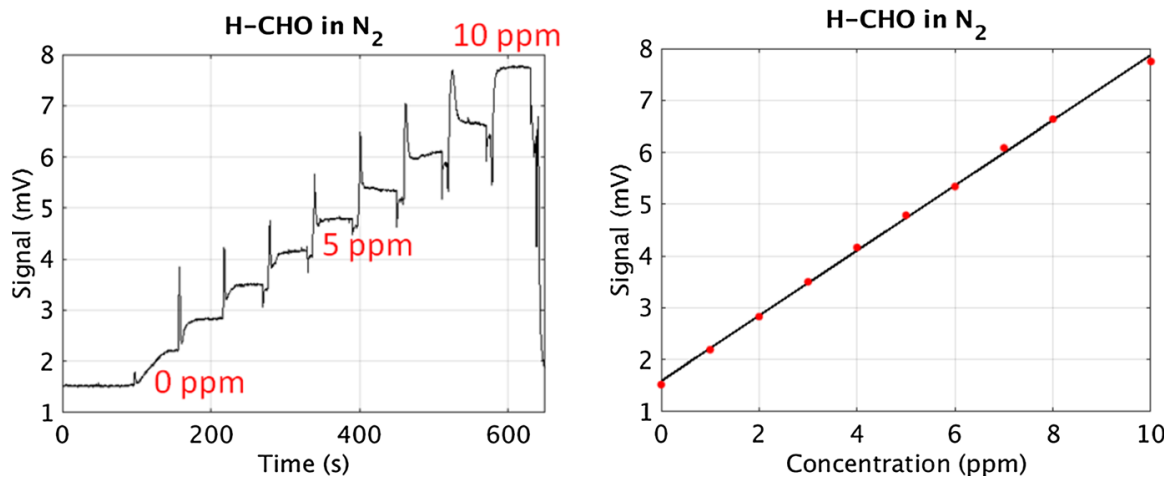

Fig. 4 Measurements for formaldehyde in nitrogen. Left figure: 1-f signal during a concentration range measurement. Right figure: linear fit for LOD determination

out 1-f and 2-f detection schemes. The integration time was set to $900 \mathrm{~ms}$ and, in both cases, based on a linear fit of the concentration range measurements, the $\mathrm{LOD}_{1 \sigma}$ was determined at $\approx 25 \mathrm{ppb}$.

Finally, a variant of the PA cell has been built in PMMA, with a geometry slightly adapted to match the lower resolution of the 3D-printing hardware (same overall dimensions but $1.5 \mathrm{~mm}$ diameter for all chambers and capillaries). The resonance frequency is $4.5 \mathrm{kHz}$ (vs. $4.2 \mathrm{kHz}$ obtained from the FEM model) and the quality factor is $\approx 3.2$. The cell was equipped with silicon windows covered with a silicon nitride antireflective coating. The results of experiments performed on a range of nitric oxide mixtures in nitrogen are presented on Fig. 5. Since then, we used a GasMix commercial diluter (AlyTech, Juvisy-sur-Orge, France) to prepare the gas samples. The light source was a QCL with $100 \mathrm{~mW}$ optical power at $1906 \mathrm{~cm}^{-1}$ (Thorlabs, Newton, NJ, USA). A 2-f detection scheme and a short integration time of $0.2 \mathrm{~s}$ led to a $\mathrm{LOD}_{1 \sigma} \approx 650 \mathrm{ppb}$, estimated from a linear fit of the measured data (Fig. 5
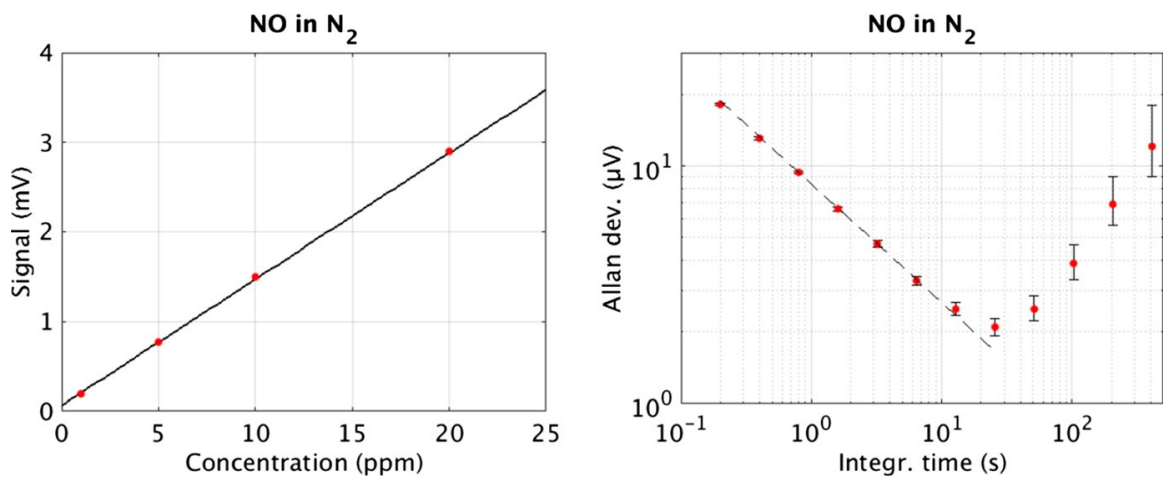

Fig. 5 Measurements performed on NO samples with the PMMA cell. Signal linearity (left) and Allan deviation (right). The dashed line represents the fit by $1 / \sqrt{\tau}$, typical of a Gaussian white noise 
left). A basic correction for the much larger volume $\left(152 \mathrm{~mm}^{3}\right.$ vs. $\left.40 \mathrm{~mm}^{3}\right)$ and much shorter integration time $(0.2 \mathrm{~s}$ vs. $7 \mathrm{~s})$ leads to a LOD with slightly reduced performances than that of the initial metal cell.

Allan deviation plot provides information about the temporal stability of the experimental set-up as a whole [18, 19]. It presents the signal standard deviation as a function of the integration time $\tau$ and allows assessing the optimal integration time and thus the ultimate detection limit of a system. Indeed, two regimes can be noticed on the graph of Fig. 5 right: the first one, where the instrument is stable and noise decreases as $1 / \sqrt{\tau}$ (typical of predominant white noise source), until an optimum, marking the occurrence of a second drift regime, where longer averaging becomes detrimental. Many sources of drift can be identified as, for instance, QCL instability, thermal drift, etc. Here, we observe a signal stability up to $\approx 25 \mathrm{~s}$ integration time and an associated ultimate $\operatorname{LOD}_{1 \sigma} \approx 15 \mathrm{ppb}$.

\subsection{Microfabricated Silicon Cell}

The same DHR architecture, still using commercial microphones, has then been employed to create the second-generation device [14]. Ten times smaller in volume than the previous one (Fig. 1), the PA cell has been produced from two silicon wafers patterned, etched, and bonded by the means of techniques commonly used in MEMS foundries. The layout has been adapted in order to match the specific constraints of microfabrication, such as for instance vertical etching in planar silicon substrates and limited depth-to-width aspect ratio (Fig. 6).

To design the miniaturized cells, we relied on the acoustic model mentioned in the previous section. However, the lengthy resolution of the FLNS equation system by the FEM can hardly be used directly within a numerical optimization algorithm, which requires multiple evaluations of the "black-box" simulator. Thus, in order to limit the use of computational resources by the optimization process, we decided to resort to the use of metamodels [20].
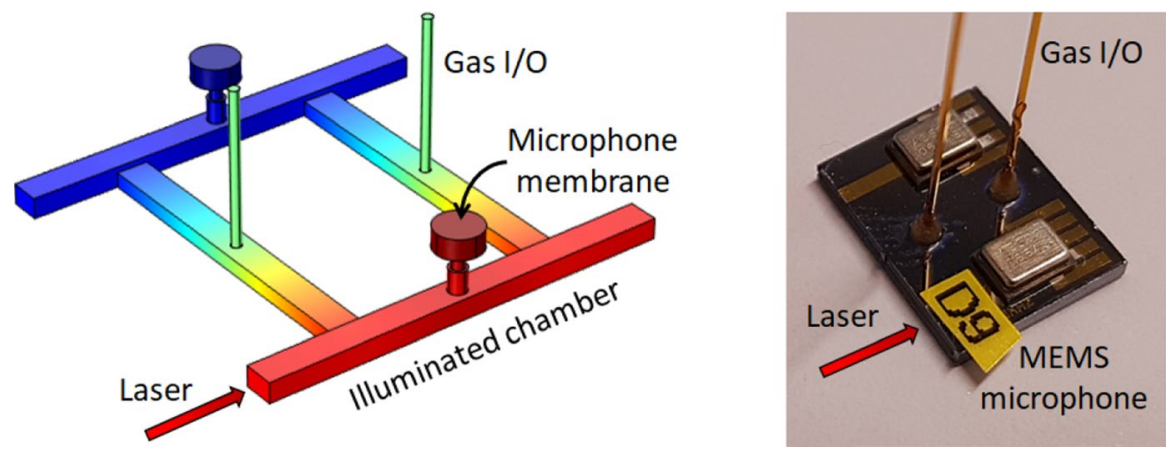

Fig. 6 Microfabricated silicon sensor. Sketch of the inner volume of the cell as used by the FEM computation (left) and picture of the sensor equipped with MEMS microphones and gas supply capillaries (right) 
The process of metamodeling successively implies evaluating the high-fidelity FEM model at points carefully chosen in the input parameter space (design of experiment) and then training the metamodel on the obtained dataset. Once the metamodel is built, the response at a new evaluation point can be predicted instantaneously. The metamodel can thus be used as a surrogate of the expensive simulator in costly procedures, such as inversion or optimization. Kriging metamodels, based on the theory of Gaussian processes, and known for their efficiency and adaptability, have been used in this work. In addition, the efficient global optimization adaptive algorithm [21], that improves the accuracy of the metamodel during the optimization by adding new points to the initial design of experiment, has been implemented.

The design problem has been posed as a constrained optimization problem: find the set of geometrical parameters maximizing the differential signal while keeping the cell resonance frequency under a given upper bound. The length of the chamber, which governs the overall size of the photoacoustic cell, as well as its cross-section, which is imposed by the angular divergence and location of the laser source, are fixed a priori by the designer. Only four dimensions are thus left in the parameter space, namely the distance between the chambers and between the capillaries, and the height and width of the capillaries. The free software toolbox SBDO, available at https://github.com/freeSBDO/SBDOT, allowed an easy implementation of the constrained optimization problem in MATLAB (The MathWorks Inc., Natick, U.S.A.).

Four configurations, corresponding to different chamber lengths $(5 \mathrm{~mm}$ and $8 \mathrm{~mm})$ and resonance frequencies $(10 \mathrm{kHz}$ and $15 \mathrm{kHz})$, have been obtained. As can be seen on Fig. 7 (left), the expected signal produced by the novel silicon cells is slightly larger than that of the 3D-printed metal cell, while still keeping a resonance frequency sufficiently low to allow for the relaxation of the molecules of interest.

Two $550 \mu \mathrm{m}$ thick silicon wafers were used to create the PA cell. The key points of the fabrication, carried out in our $200 \mathrm{~mm}$ MEMS production facility, consisted in (i) etching, in both wafers, the deep trenches constituting the chambers and capillaries and (ii) airtight molecular bonding of the two wafers. Metal contacts were patterned on the upper wafer in order to allow soldering the microphones and
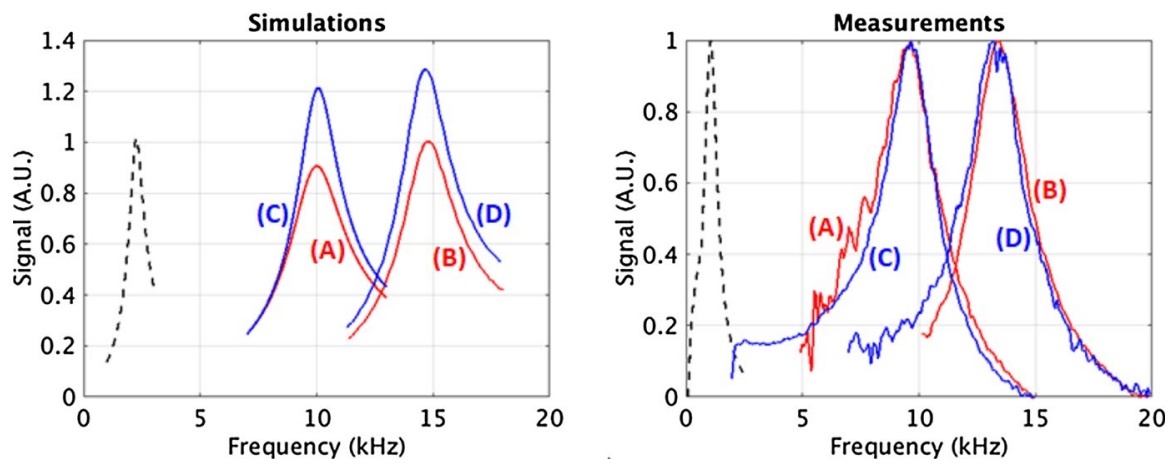

Fig. 7 Computed (left) and measured (right) resonance spectra of the DHR-based PA cells. The red and blue lines represent the four variants (A to D) of the microfabricated silicon cell and the black dashed line represents the $3 \mathrm{D}$-printed metal cell 
outputting the electrical signal. After soldering the two analogical AKU350 MEMS microphones (Akustika, Bosch Germany) and dicing, the PA chip was mounted on a flexible PCB and the electrical contacts were wire-bonded. Finally, fused silica capillaries were connected to the gas inlet and outlet to provide calibrated gas samples.

A specialized electronic board, including a digital lock-in amplifier and signal processing functions, was developed at this occasion to insure a high signal-to-noise ratio and to guarantee the consistency of the miniaturization of the sensor and of its associated electronics.

In a first step, the resonance frequency of the silicon cells was measured using a $5.5 \mathrm{~mW}$ distributed feedback QCL from mirSense (Palaiseau, France), targeted at the $2302 \mathrm{~cm}^{-1}$ carbon dioxide absorption line. Measurements were performed directly on ambient air. As can be seen on Fig. 7, all four variants resonate close to the expected resonance frequencies (maximum discrepancy is less than $9 \%$ ) and the values of the measured quality factors are comparable to the computed ones (2.2 to 3.3 vs. 2.5 to 3.9$)$.

The microfabricated silicon cells have then been thoroughly characterized and the main operating parameters, such as LOD, stability, and NNEA, have been determined in different conditions of operation.

The first series of experiments presented here used a mixture of carbon monoxide in nitrogen. The continuous wave QCL (Alpes laser, Neuchâtel, Switzerland) emitted $75 \mathrm{~mW}$ at $2127.7 \mathrm{~cm}^{-1}$. Both 1-f and 2-f wavelength modulation schemes were used (Fig. 8) and the $\mathrm{LOD}_{1 \sigma}$ reached with $2.1 \mathrm{~s}$ integration time was, respectively, estimated at $\approx 420 \mathrm{ppb}$ and $\approx 450 \mathrm{ppb}$.

A second series of measurements was carried out on nitric oxide with the experimental set-up described previously for the 3D-printed PMMA cell. This time, we were interested in the spectroscopic capabilities of the cell. Several peaks corresponding to the absorption of water $(\approx 7000 \mathrm{ppm})$ and nitric oxide $(20 \mathrm{ppm})$ can clearly be seen on the left part of Fig. 9. A concentration range of nitric oxide in nitrogen was also measured with a QCL optical power of $123 \mathrm{~mW}$ at $1903.1 \mathrm{~cm}^{-1}$ (Fig. 9, right) and using a 2-f modulation scheme. Let us note that the signal returns
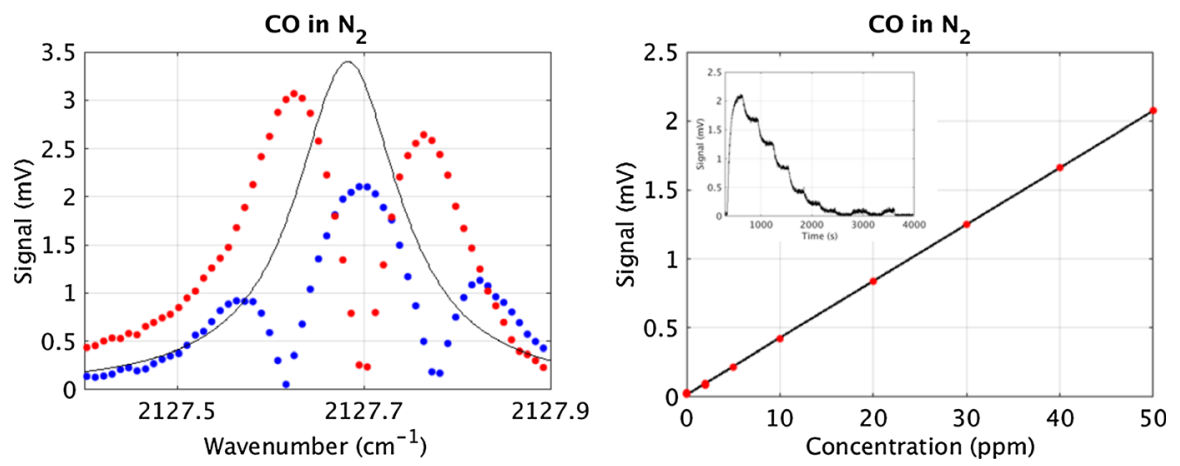

Fig. 8 Measurements of carbon monoxide in nitrogen. Left figure: 1-f (red) and 2-f (blue) spectra, superimposed with the targeted absorption peak (solid line). Right figure: linear fit of the 2-f signal vs. concentration. Concentration range $(50 \mathrm{ppm}$ to $0 \mathrm{ppm})$ presented in inset 

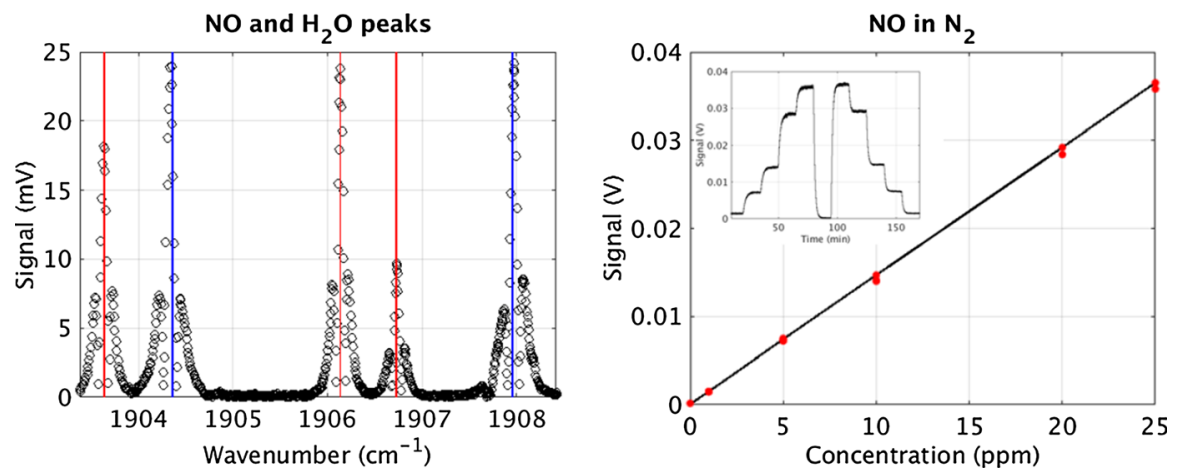

Fig. 9 Measurements of nitric oxide. Left figure: measured 2-f spectrum (o) superimposed with the lines of $\mathrm{NO}$ (red) and $\mathrm{H}_{2} \mathrm{O}$ (blue). Right figure: linear fit of the 2-f signal vs. concentration, with concentration range ( $1 \mathrm{ppm}$ to $25 \mathrm{ppm}$ ) presented in inset

to zero after several increasing or decreasing concentration steps. This suggests that surface adsorption is negligible. Here, we obtained a $\operatorname{LOD}_{1 \sigma} \approx 89 \mathrm{ppb}$.

In parallel, measurements, presented on Fig. 10, were conducted to assess the signal stability of the two set-ups used for the detection of carbon monoxide (blue dots) and nitric oxide (green dots). Both Allan deviation plots show a similar behavior, with a maximum integration time larger than $10 \mathrm{~s}$. Regarding nitric oxide, the ultimate $\mathrm{LOD}_{1 \sigma}$ is around $9 \mathrm{ppb}$ for a $12 \mathrm{~s}$ averaging time. Let us note that, when compared with the experiments performed with the 3D-printed PMMA cell in similar conditions (same QCL), the level of noise is increased by almost an order of magnitude.

A last set of experiments was performed on a mixture of fluorocarbon in nitrogen with a silicon cell thermally regulated at $25{ }^{\circ} \mathrm{C}$. The QCL (Alpes laser, Neuchâtel, Switzerland) emitted $67 \mathrm{~mW}$ at $1283 \mathrm{~cm}^{-1}$. A 2-f wavelength modulation scheme was used and the $\operatorname{LOD}_{1 \sigma}$ was estimated at $\approx 18 \mathrm{ppb}$ with an integration time of $1 \mathrm{~s}$. In order to identify some sources of drift, Allan deviation plots are presented on

Fig. 10 Allan deviation plots corresponding to the microfabricated silicon cell (NO, green and $\mathrm{CO}$, blue) and the 3D-printed PMMA cell (red: NO) (Color figure online)

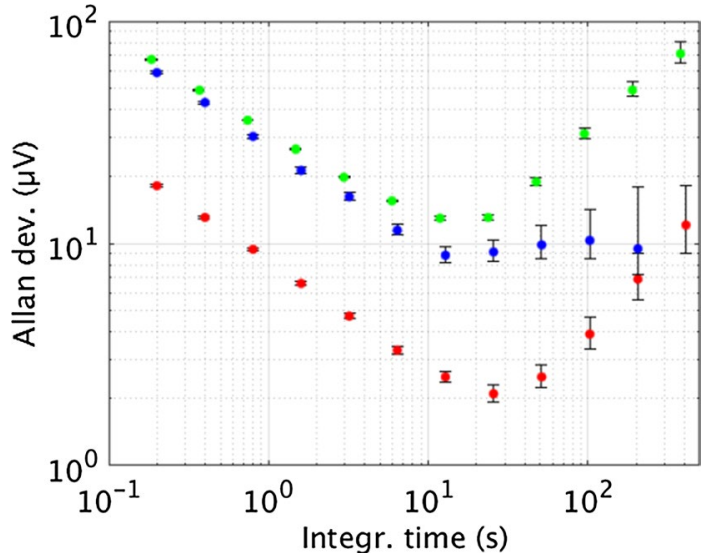


Fig. 11 for three different experimental configurations, respectively, with the laser off, with the laser on in air, and with the laser on and gas mixture present. As can be seen, switching on the laser (blue dots) adds a significant level of white noise and constitutes a first source of drift, with a stability up to $\approx 1000 \mathrm{~s}$. With the gas of interest present (green dots), the system is still quite stable, with an optimal integration time $\approx 50 \mathrm{~s}$ and an ultimate $\mathrm{LOD}_{1 \sigma} \approx 2 \mathrm{ppb}$.

\section{Silicon-integrated Photoacoustic Trace Gas Sensor}

As the chamber volume decreases, the acoustic resonance frequency increases and the influence of the viscous and thermal surface losses effects becomes prominent. Thus, further miniaturization of classical photoacoustic cells architectures faces fundamental restrictions.

Firstly, due to the relaxation dynamics of the gas molecules involved in the signal generation, it is necessary to keep the laser modulation frequency sufficiently low to guarantee proper heat relaxation, and thus pressure fluctuation production. Depending on the molecule of interest and the gas matrix, the relaxation time constant can vary on several orders of magnitude. Thus, in order to retain the possibility of detecting several different species with the same design of PA cell, it is advisable to fix an upper limit around $20 \mathrm{kHz}$ [3].

Secondly, the signal amplification is generally obtained by tuning the modulation frequency with the resonance frequency of the acoustic network [2]. If, indeed, a differential Helmholtz architecture can be used to lower the resonance frequency of the acoustic network, the amplification obtained is still limited by the gas viscosity, which, in small chambers, drastically deteriorates the quality factor of such acoustic resonator (quality factor values around a few units).

Finally, commercial MEMS microphones are primarily designed to cover the entire acoustic frequency range and to detect acoustic pressure fluctuations in the open field [22]. They generally have an expansion volume larger than $3 \mathrm{~mm}^{3}$. Therefore, the operating point of the microphone acoustic network is drastically affected

Fig. 11 Allan deviation plot for $\mathrm{CF}_{4}$ in nitrogen. Measurements with laser off (red), laser on in air (blue), laser on and $2 \mathrm{ppm}$ $\mathrm{CF}_{4}$ in nitrogen (green) (Color figure online)

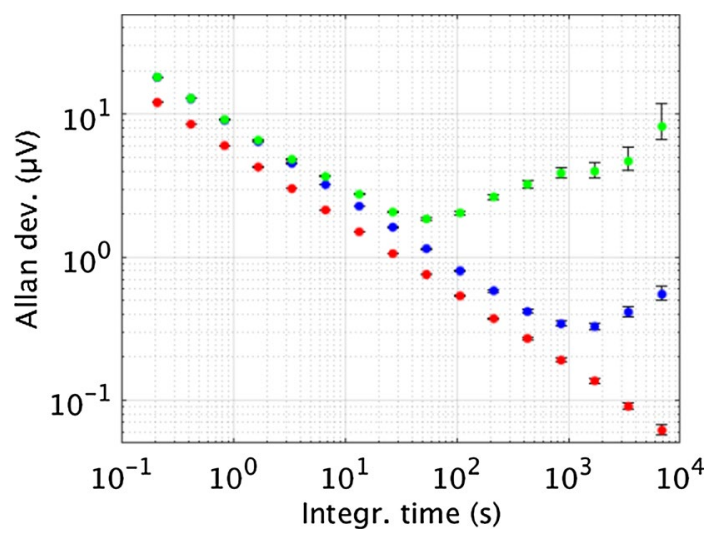


when the microphone is connected to the small and closed front volume constituted by the PA cell.

\subsection{Design}

In order to overcome these limitations, a completely different architecture has been devised for the third generation device, which includes its own dynamic pressure sensor [15]. The cell consists in an illuminated chamber, separated from a closed expansion volume by a channel where a cantilever beam is placed (Fig. 12). Here, the laser modulation is tuned to the mechanical resonance of the cantilever and not, as usually, to the acoustic resonance of the cell.

This option is beneficial regarding the resonance frequency, which can be kept by design in the $\mathrm{kHz}$ range, and the quality factor. In addition, other advantages can be highlighted. Firstly, the mechanical resonance is less prone to variations due to the environmental conditions (pressure, temperature, change in gas matrix, etc.) than the acoustic resonance. Secondly, this architecture is inherently sensitive to pressure difference between the illuminated chamber and the closed expansion volume in the acoustic network. Thus, no microphone matching is needed as in the former DHR cells. Thirdly, we kept a symmetric arrangement for the fluidic network in such a way that external perturbations arising at the inlet or the outlet result mainly in a common mode pressure fluctuation in the acoustic network. As can be seen on Figs. 12 and 15, the fluidic capillaries used to connect gas inlet or outlet to the chambers of the acoustic network are identical.

The operating principle of the sensor is as follows (Fig. 13). The modulated illumination establishes a pressure fluctuation in the PA chamber. The dynamic pressure difference arising between the PA chamber and the passive expansion volume makes the cantilever beam rotate around a flexible hinge, in the plane of the wafer. The longitudinal stress induced within doped silicon nanogauges by the motion of the cantilever causes an electrical resistance variation through piezoresistive effect. This resistance variation is proportional to the pressure difference and thus to the concentration of the molecule of interest.
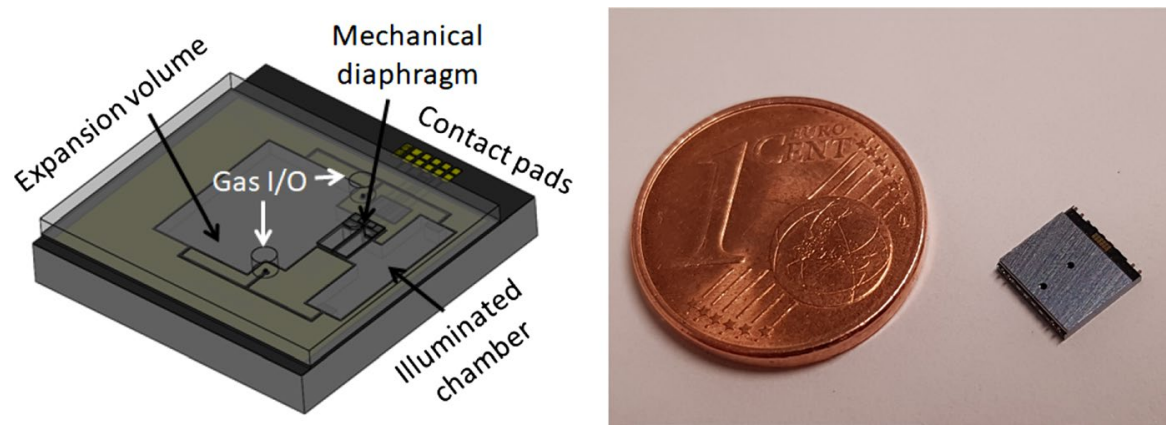

Fig. 12 Drawing of the silicon-integrated photoacoustic sensor (left) and view of the manufactured diced chip (right) 


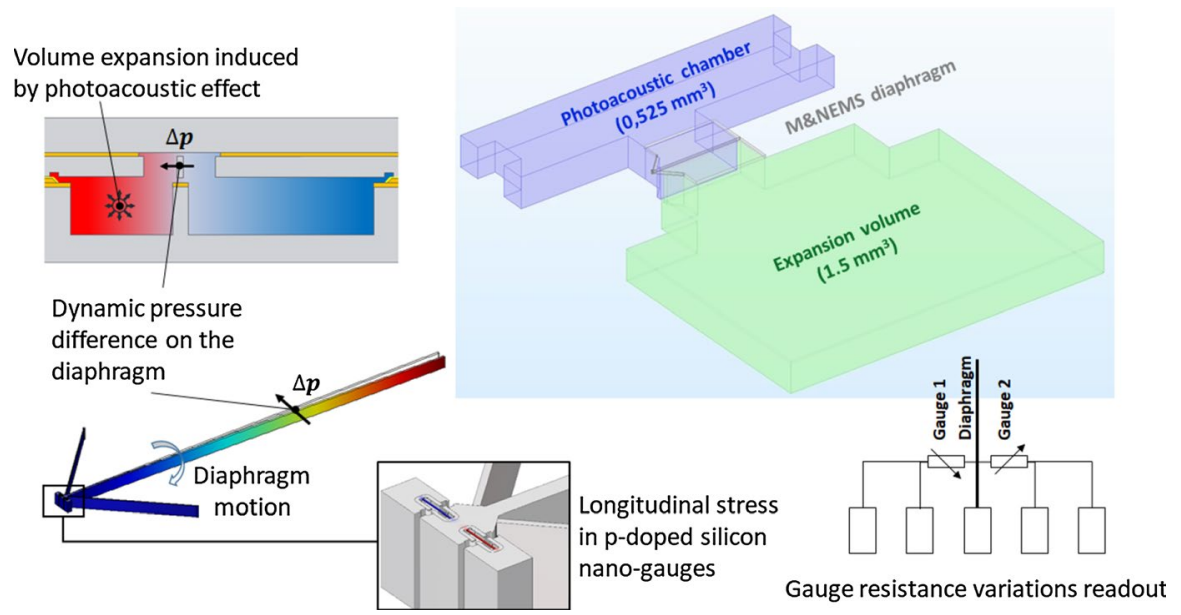

Fig. 13 Physical processes involved in the measurement chain: from thermal relaxation of excited molecules to resistance variation readout

The efficiency of the measurement chain is linked to the strong amplification of the stress level in the gauges (the signal is amplified before being degraded by the electrical noise from the gauges). This stress amplification is provided by two mechanical means: firstly by the contrast between the lateral surface of the cantilever beam and the section of the nanogauges, and secondly by a lever effect involving the ratio between the cantilever's length over the hinge-to-gauges' distance.

The design of this MEMS sensor requires an accurate model, coupling the photoacoustic, acoustic, mechanical, and electrical behaviors of the system. A complete lumped model, based on the electromechanical analogy has been devised and described previously [15]. The $3.9 \mu \mathrm{m}$ high by $750 \mu \mathrm{m}$ long cantilever beam has been designed to resonate at $5.33 \mathrm{kHz}$ in free space. When installed in the channel, due to the contrast between the compliances of the cantilever and the acoustic network, the calculated mechanical resonance frequency shifts to $6.5 \mathrm{kHz}$.

The overall footprint of the diced device is $5.5 \mathrm{~mm} \times 5.5 \mathrm{~mm}$, with an inner volume of $2.3 \mathrm{~mm}^{3}$.

\subsection{Fabrication}

The PA detectors were fabricated in the CEA-LETI $200 \mathrm{~mm}$ silicon facilities by stacking two wafers (Fig. 14). The so-called "M\&NEMS" technological process [23] was applied to the first wafer, called "Sensor" wafer. This wafer comprises the mechanical cantilever diaphragm and the piezoresistive gauges (in p-doped silicon), as well as the capillaries used to connect the fluidic ports to the acoustic network. The second "Cap" wafer includes the PA chamber, the expansion volume, the electrical routing, and contacts. These wafers are sealed together using gold-silicon eutectic bonding. Grinding process and deep reactive ion etching are then used to thin the Sensor wafer down to $300 \mu \mathrm{m}$ thickness and produce the fluidic ports 


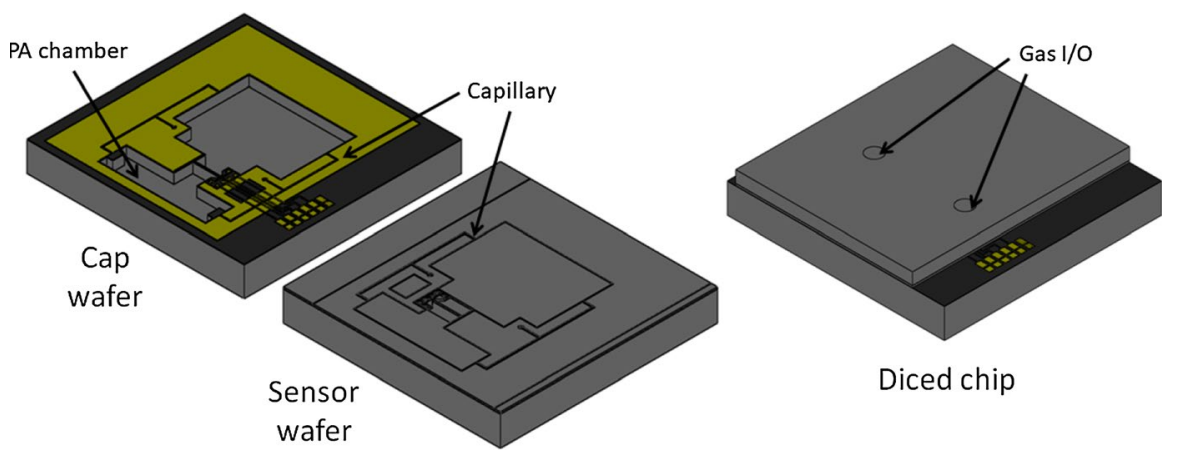

Fig. 14 Drawing of the two wafers stack used to build the integrated PA sensor (not to scale)

required to fill the acoustic network with the gas. The sealed wafer is finally partially diced to reveal the electric contacts (saw to reveal method).

Figure 15 presents inner views of the device obtained at intermediate stages of the fabrication. The scanning electron microscope view on the left demonstrates the excellent quality of the realization of the micromachined cantilever beam. Let us also note that full wafer characterization of microphones produced on the same batch showed that $65 \%$ of the devices pass acoustic tests. Obtaining this yield value on a first run is of course very promising regarding the potential of industrialization of the process.

\subsection{Measurements}

To begin with, the response of the cell as a function of the modulation frequency of the laser was determined experimentally. The measurement was carried out on ambient air, using the carbon dioxide absorption line located at the optical wavenumber of $2302.7 \mathrm{~cm}^{-1}$. Figure 16 presents the excellent agreement of the theoretical readout signal, provided by the lumped model, and the measured signal. A
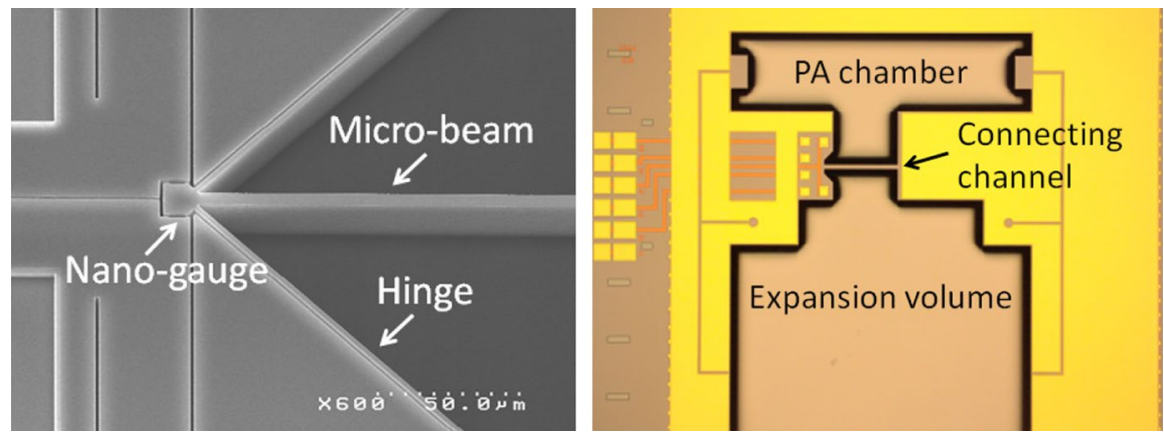

Fig. 15 Scanning electron microscope view of the micromachined cantilever beam close to its anchoring point (left) and optical microscope photograph of the cap wafer (right) 

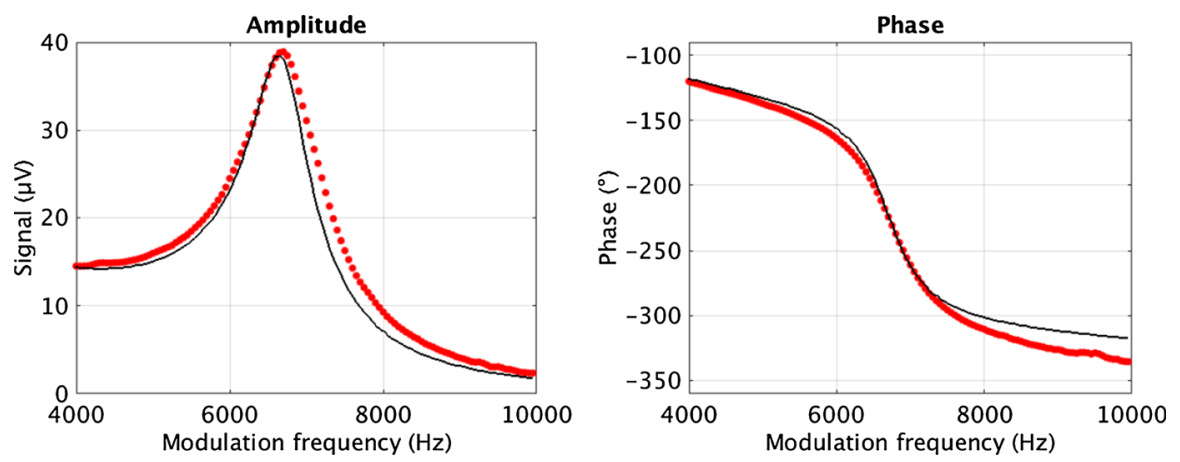

Fig. 16 Frequency response of the PA sensor. Comparison between simulated (solid line) and measured (dotted line) values of the signal amplitude and phase

fit of the experimental transfer function provides a first estimation $(Q \approx 10)$ of the mechanical quality factor in ambient air.

Further measurements were performed to assess the gas-sensing capabilities of the device. An interband cascade laser (Nanoplus, Gerbrunn, Germany) tuned to the $2979 \mathrm{~cm}^{-1}$ absorption line of methane was focused into the photoacoustic chamber of the PA cell while injecting a methane and nitrogen gas mixture through its fluidic port. The laser was wavelength-modulated at the mechanical resonance frequency of the cantilever beam $\mathrm{f}_{0}$ (respectively $\mathrm{f}_{0} / 2$ ), and a $1-\mathrm{f}$ (respectively, 2-f) detection scheme was applied to retrieve the photoacoustic signal presented on the left part of Fig. 17. The relatively complex line structure of methane in the range of wavenumbers $^{1}$ is satisfactorily rendered by the 1-f and 2-f profiles, although the convolution is too pronounced to resolve the two main absorption peaks. The limit of detection of the sensing chain is estimated at $\mathrm{LOD}_{1 \sigma} \approx 15 \mathrm{ppm}$ for a relatively short integration time of $0.21 \mathrm{~s}$. Allan deviation measurement presented in the right part of Fig. 17 shows that the long-term stability of the detection chain is good, reaching
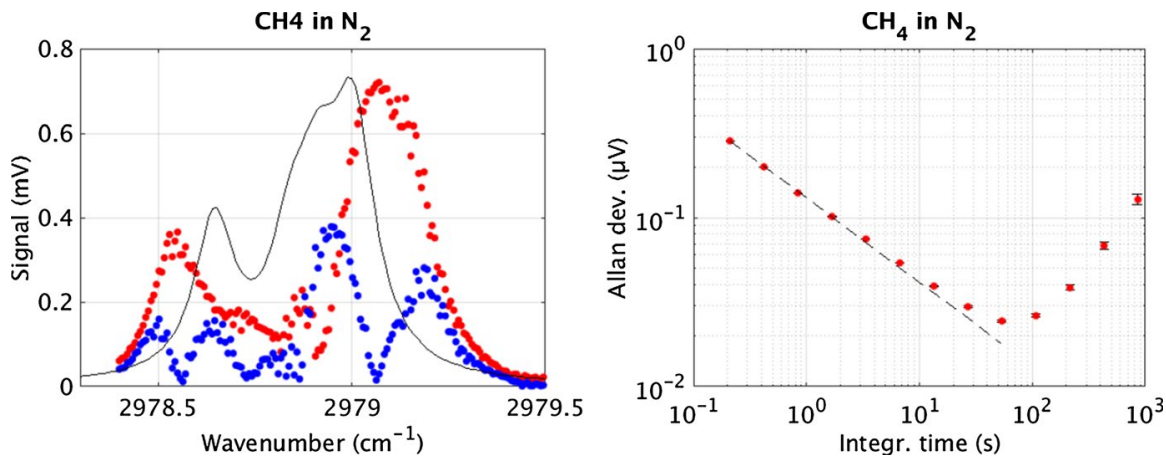

Fig. 17 Measurements of methane in nitrogen. Left figure: 1-f (red) and 2-f (blue) spectra, superimposed with the targeted absorption peak (solid line). The measured values were shifted by $0.13 \mathrm{~cm}^{-1}$. Right figure: Allan deviation plot. The dashed line represents the fit by $1 / \sqrt{\tau}$ 
its optimal performance for a $54 \mathrm{~s}$ integration time. The corresponding ultimate limit of detection is estimated at $\mathrm{LOD}_{1 \sigma} \approx 1.4 \mathrm{ppm}$. Similar measurements carried out on the same devices with a QCL aimed at the $2302 \mathrm{~cm}^{-1}$ carbon dioxide absorption line led to a $\mathrm{LOD}_{1 \sigma}$ of $0.9 \mathrm{ppm}$ for a $7 \mathrm{~s}$ integration time.

\section{Summary and Perspectives}

In summary, three small-scale PA cells $\left(40 \mathrm{~mm}^{3}\right.$ to $\left.2.3 \mathrm{~mm}^{3}\right)$, made of steel or silicon, based on two different architectures have been designed, fabricated, and tested (Fig. 18). All three versions reach sub-ppm limit of detection but each has its own benefits and drawbacks, regarding fabrication, implementation, and ease of use. For instance, the 3D-printed cell is well adapted for rapid prototyping and benchtop testing, whereas the microfabricated silicon cell would be a better fit for industrial series involving pick-and-place soldering of the MEMS microphones and advanced packaging.

The third-generation component is far more demanding in terms of fabrication cycle time and technological means involved but could ultimately be produced at high-volume and at a negligible cost. Moreover, its small volume allows a simple filling, by diffusion, without a pumping system. The sensor can thus be integrated more easily in a portable system. An improved version is currently under fabrication. Among the expected benefits, let us emphasize an easier gas supply, with an improved fluidic circuit and I/O ports implemented on the backside, and a fully CMOS compatible bonding method.

Two figures of merit, namely LOD and NNEA, have been chosen to summarize the performances of the PA cells placed in different measurement conditions (Table 1). These figures depend significantly on the whole experimental set-up, and not only on the PA cell. They nevertheless constitute a helpful cross-comparison tool. The LOD is calculated with signal and noise values measured with the integration time specified in the Table. It is greatly variable, in particular because of differences in available laser power, peak intensity, and integration time but all, except for the last one, are in the sub-pm range. The NNEA also differs notably between the cells and experiments, with however a consistently better performance obtained with

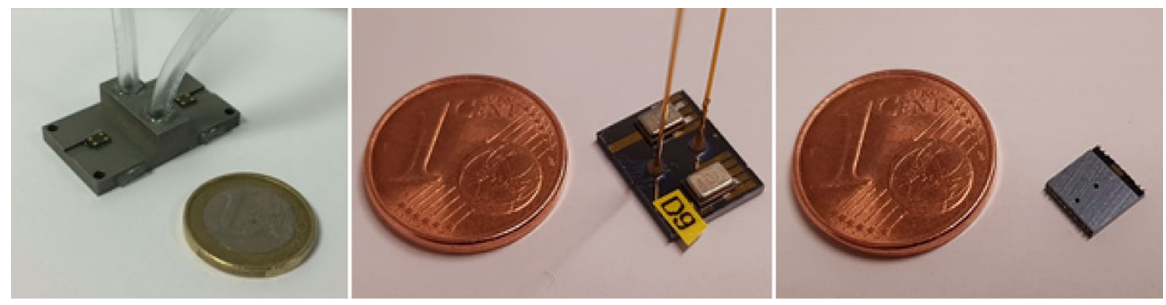

Fig. 18 Three generations of miniaturized PA cells. From left to right, the 3D-printed metal cell, the microfabricated silicon cell, and the silicon cell with its built-in piezoresistive pressure sensor 
the larger metal cell, in the $10^{-8} \cdot \mathrm{cm}^{-1} \cdot \mathrm{W} / \mathrm{Hz}^{1 / 2}$ range. In this paper, the initial definition by Kosterev et al. [24] is used and we define the NNEA as

$$
N N E A_{1 \sigma}=\frac{\alpha P_{o p t}}{S N R \sqrt{\Delta f}},
$$

where $\alpha$ is the gas absorption coefficient at the considered absorption line $\left(\mathrm{cm}^{-1}\right)$, $\mathrm{P}_{\text {opt }}$ is the optical power of the laser $(\mathrm{W}), \mathrm{SNR}$ is the $1 \sigma$ signal-to-noise ratio, and $\Delta \mathrm{f}$ is the equivalent noise bandwidth of the lock-in amplifier $(\mathrm{Hz})$. Let us note that, in all the experiments, the value used for $\mathrm{P}_{\mathrm{opt}}$ is the optical power at the laser output, instead of the power really injected in the chamber. Thus, all the values of NNEA provided are most probably overestimated.

In parallel to this work on PA cells, significant progress has also been made in our institute towards the wafer scale fabrication of quantum cascade lasers [25] and adapted photonic integrated circuits [26]. It thus seems that the goal initially stated, namely to build a PA sensor combining, on the same chip, a mid-infrared laser, a light injection circuit, and a PA cell including an integrated pressure sensor is now within reach $[27,28]$.

Acknowledgements The authors are indebted to all the members of the teams running the CEA-LETI $200 \mathrm{~mm}$ cleanrooms facilities where all silicon cells were fabricated and to Nadine David, in charge of packaging. They also acknowledge the valuable contributions by colleagues from GSMA, Univ. of Reims Champagne Ardenne, France (B. Parvitte, V. Zéninari), Fraunhofer IPM Freiburg (T. Strahl), and at mirSense (G.Aoust, F. Boulila, M. Brun, and M. Carras). The funding of this work has been in part provided by national grants (MIRIADE, ANR-11-ECOT-04) or European grants (MIRIFISENS, FP7 317884, REDFINCH, H2020 780240) and the MIRPHAB consortium, as well as by several industrial partners (currently mirSense, in the framework of our joint laboratory). All Allan deviation plots presented in this paper were calculated with the ALAVAR software: thanks to the author, Alaa Makdissi, for making it freely available at http://www.alamath.com/alavar.

Open Access This article is licensed under a Creative Commons Attribution 4.0 International License, which permits use, sharing, adaptation, distribution and reproduction in any medium or format, as long as you give appropriate credit to the original author(s) and the source, provide a link to the Creative Commons licence, and indicate if changes were made. The images or other third party material in this article are included in the article's Creative Commons licence, unless indicated otherwise in a credit line to the material. If material is not included in the article's Creative Commons licence and your intended use is not permitted by statutory regulation or exceeds the permitted use, you will need to obtain permission directly from the copyright holder. To view a copy of this licence, visit http://creativecommons.org/licen ses/by/4.0/.

\section{References}

1. R. Bogue, Sens. Rev. 33, 300-304 (2013)

2. A. Miklos, P. Hess, Z. Bozoki, Rev. Sci. Instrum. 72, 1937 (2001)

3. A. Glière, J. Rouxel, M. Brun, B. Parvitte, V. Zéninari, S. Nicoletti, Sensors 14, 957 (2014)

4. D. Popa, F. Udrea, Sensors 19, 2076 (2019)

5. R. Bauer, G. Stewart, W. Johnstone, E. Boyd, M. Lengden, Opt. Lett. 39, 4796 (2014)

6. T. Rück, R. Bierl, F.-M. Matysik, Sens. Actuators A 263, 501 (2017)

7. V. Wittstock, L. Scholz, B. Bierer, A.O. Perez, J. Wöllenstein, S. Palzer, Sens. Actuators B Chem. 247, 930 (2017) 
8. M. Weber, P. Lerch, P. Renaud, in Simulation and Design of Microsystems and Microstructures II, ed. by R. Adey, P. Renaud (WIT Press, Southampton, 1997), pp. 117-126

9. N. Ledermann, P. Muralt, J. Baborowski, M. Forster, J.-P. Pellaux, J. Micromech. Microeng. 14, 1650 (2004)

10. S.L. Firebaugh, K.F. Jensen, M.A. Schmidt, J. Microelectromech. Syst. 10, 232 (2001)

11. P.M. Pellegrino, R.G. Polcawich, S. L. Firebaugh, in Chemical and biological sensing V, ed. by P.J. Gardner (Spie-Int Soc Optical Engineering, Bellingham, 2004), pp. 42-53

12. E.L. Holthoff, D.A. Heaps, P.M. Pellegrino, IEEE Sens. J. 10, 572 (2010)

13. J. Rouxel, J.-G. Coutard, S. Gidon, O. Lartigue, S. Nicoletti, B. Parvitte, R. Vallon, V. Zéninari, A. Glière, Sens. Actuators B Chem. 236, 1104 (2016)

14. J.-G. Coutard, A. Glière, J.-M. Fedeli, O. Lartigue, J. Skubich, G. Aoust, A. Teulle, T. Strahl, S. Nicoletti, M. Carras, L. Duraffourg, in MOEMS and Miniaturized Systems XVIII (International Society for Optics and Photonics, San Francisco, USA, Feb. 02-07, 2019), p. 109310V

15. H. Lhermet, T. Verdot, A. Teulle, A. Berthelot, A. Glière, B. Desloges, F. Souchon, M. Fournier, J.-M. Fedeli, J.-G. Coutard, in Transducers 2019 (Berlin, Germany, Jun. 23-27, 2019)

16. V. Zeninari, V.A. Kapitanov, D. Courtois, Y.N. Ponomarev, Infrared Phys. Technol. 40, 1 (1999)

17. A. Glière, J. Rouxel, B. Parvitte, S. Boutami, V. Zéninari, Int. J. Thermophys. 34, 2119 (2013)

18. P. Werle, R. Mücke, F. Slemr, Appl. Phys. B 57, 131 (1993)

19. M. Giglio, P. Patimisco, A. Sampaolo, G. Scamarcio, F.K. Tittel, V. Spagnolo, IEEE Trans. Ultrason. Ferroelect. Freq. Contr. 63, 555 (2016)

20. A. Forrester, D.A. Sobester, A. Keane, Engineering Design via Surrogate Modelling: A Practical Guide (Wiley, Hoboken, 2008)

21. D.R. Jones, M. Schonlau, W.J. Welch, J. Glob. Optim. 13, 455 (1998)

22. A. Dehe, M. Wurzer, M. Fuldner, U. Krumbein, in AMA Conferences 2013, SENSOR 2013 (Nürnberg, Germany, May 14-16, 2013), pp. 95-99

23. P. Robert, V. Nguyen, S. Hentz, L. Duraffourg, G. Jourdan, J. Arcamone, S. Harrisson, in Proceedings of the IEEE Sensors 2009 Conference (Christchurch, New Zealand, Oct. 25-28, 2009), pp. 963-966

24. A.A. Kosterev, F.K. Tittel, D.V. Serebryakov, A.L. Malinovsky, I.V. Morozov, Rev. Sci. Instrum. 76, 043105 (2005)

25. J.-G. Coutard, M. Brun, M. Fournier, O. Lartigue, F. Fedeli, G. Maisons, J.-M. Fedeli, S. Nicoletti, M. Carras, and L. Duraffourg, Submitted to Sci. Rep. (n.d.)

26. P. Barritault, M. Brun, P. Labeye, J.-M. Hartmann, F. Boulila, M. Carras, S. Nicoletti, Opt. Express 23, 26168 (2015)

27. S. Nicoletti, P. Barritault, S. Boutami, M. Brun, A. Glière, P. Labeye, J. Rouxel, J. Czarny, H. Lhermet, M. Carras, and G. Maisons, in IEEE Sensors 2014 Conference (Valencia, Spain, Nov. 2-5, 2014)

28. S. Nicoletti, J.-M. Fédéli, M. Fournier, P. Labeye, P. Barritault, A. Marchant, A. Glière, A. Teulle, J.-G. Coutard, and L. Duraffourg, in Silicon Photonics XIV (International Society for Optics and Photonics, San Francisco, USA, Feb. 02-07, 2019), p. 109230H

Publisher's Note Springer Nature remains neutral with regard to jurisdictional claims in published maps and institutional affiliations. 